\title{
Training and caring for primates
}

\author{
Genevieve Andrews-Kelly, BS, LATG, Animal Care and Use Coordinator, \\ Primate Behaviorist, Envigo, Princeton, NJ
}

\begin{abstract}
Ms. Andrews-Kelly recounts the experiences and inspirations that have guided her career in providing care, enrichment and training for non-human primates.
\end{abstract}

\section{What lead you to pursue a career caring for laboratory animals?}

In college, I studied physical anthropology and non-human primates (NHPs), and I planned to go to graduate school to study primatology in the field. Through a friend, I was offered an opportunity to volunteer at a primate facility, Laboratory for Experimental Medicine and Surgery in Primates (LEMSIP). This volunteer opportunity changed the trajectory of my career plan, and I decided to pursue a career in laboratory animal science. As a volunteer, my main responsibility was to make and distribute enrichment for the chimpanzees, macaques, marmosets and tamarins. After a year at LEMSIP, I was hired as an Animal Technician. It was at LEMSIP that I learned about enrichment and the specialized needs of nonhuman primates in a captive setting. After I left LEMSIP to pursue other opportunities, I continued to volunteer at the facility for about a year. I loved spending time with the infant chimpanzees in the nursery and making enrichment for the other primates.

Since LEMSIP, I have worked in pharmaceutical companies, academic settings and contract research organizations. I have worked on various types of research, from neuroscience to veterinary technician work, but my constant throughout these various roles has been a focus on NHPs, their enrichment and their welfare.

\section{What were some of the challenges and inspirations that you encountered in your career?}

One of the most rewarding challenges was the implementation of the monkey positive reinforcement training program at Envigo. MATT (Monkey and Technician Training) was started in 2002 because of my interest in advancing primate welfare. After many revisions and updates, the MATT program is still going strong at Envigo. While the development and execution of the MATT program was an initial challenge, my manager, Dr. Terry Kusznir, believed in the importance of implementing a positive reinforcement training program and supported the launch of the program. It was a huge help, having a supportive manager who shares my interest in advancing welfare!

I am very lucky to have had great supervisors and mentors throughout my career. Cynthia Kirby lead my initial training in enrichment and taught me a lot about primate behavior and ways to support speciestypical behaviors in the laboratory setting. Jayne Mackta has also been a fantastic mentor, and through working with her I have had many opportunities to advance enrichment education and outreach for the laboratory animal community.

I believe that it is our responsibility to make every day the animals' best day. That was my inspiration at the beginning of my career, and I still believe in that.

\section{What is a typical day like for you in your current position at Envigo?}

One of my main responsibilities is to oversee our in-house colonies and allocate animals to studies. I am directly responsible for our MATT program. I train monkeys for study procedures and train our technicians on positive reinforcement techniques. Training is my favorite part of my current position, and I enjoy training the human and nonhuman primates. I am an IACUC member, I review protocols and I perform inspections and program reviews.

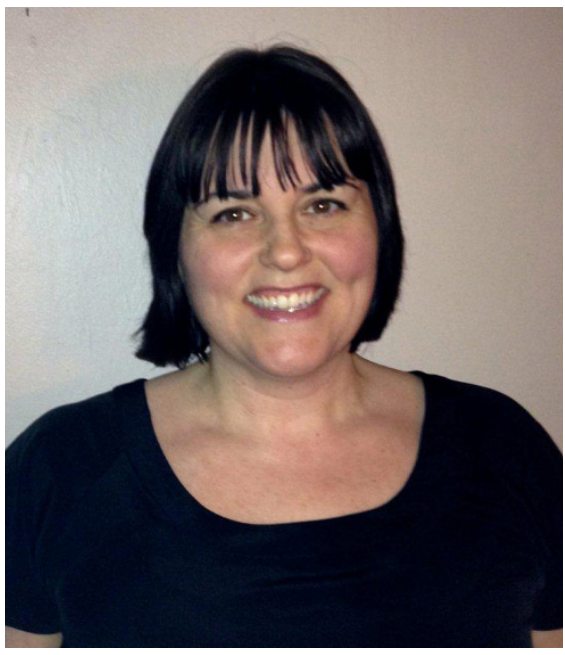

At the 2015 AALAS National Meeting you gave a wonderful talk on the importance of the human-animal bond in animal research. How has this bond helped you throughout your career?

My main motivation is spending time with the animals. My bond with the animals that I work with is ultimately my best reinforcement. I have always found it incredibly rewarding to provide for the psychological wellbeing of the animals under my care.

There are particular animals that hold a special place in my heart. Some were animals that were a challenge to train, and we developed a bond through our training sessions. Others just brightened my day. Every day I look forward to seeing our animals. They are my true motivation for being in laboratory animal science.

In my experience, the bonds that are created with our animals are critical to supporting animal welfare. In our industry, we have some unique challenges to these bonds, which can be difficult for us. We need to acknowledge these challenges in order to continue to bond with the animals in our care. Caring and compassionate laboratory animal professionals are valuable, and I believe that supporting these bonds will enable us to continue our challenging and important work. 